\title{
SCIENTIFIC REPORTS

\section{OPEN Antarctic root endophytes improve physiological performance and yield in crops under salt stress by enhanced energy production and $\mathrm{Na}^{+}$sequestration}

\author{
Marco A. Molina-Montenegro ${ }^{1,2,3^{*}}$, Ian S. Acuña-Rodríguez ${ }^{1}$, Cristian Torres-Díaz ${ }^{4}$, \\ Pedro E. Gundel ${ }^{5}$ \& Ingo Dreyer ${ }^{6}$
}

Climatic change is pointed as one of the major challenges for global food security. Based on current models of climate change, reduction in precipitations and in turn, increase in the soil salinity will be a sharp constraint for crops productivity worldwide. In this context, root fungi appear as a new strategy to improve plant ecophysiological performance and crop yield under abiotic stress. In this study, we evaluated the impact of the two fungal endophytes Penicillium brevicompactum and $P$. chrysogenum isolated from Antarctic plants on nutrients and $\mathrm{Na}^{+}$contents, net photosynthesis, water use efficiency, yield and survival in tomato and lettuce, facing salinity stress conditions. Inoculation of plant roots with fungal endophytes resulted in greater fresh and dry biomass production, and an enhanced survival rate under salt conditions. Inoculation of plants with the fungal endophytes was related with a higher up/down-regulation of ion homeostasis by enhanced expression of the NHX1 gene. The two endophytes diminished the effects of salt stress in tomato and lettuce, provoked a higher efficiency in photosynthetic energy production and an improved sequestration of $\mathrm{Na}^{+}$in vacuoles is suggested by the upregulating of the expression of vacuolar $\mathrm{NHX} 1 \mathrm{Na}^{+} / \mathrm{H}^{+}$antiporters. Promoting plant-beneficial interactions with root symbionts appears to be an environmentally friendly strategy to mitigate the impact of climate change variables on crop production.

The earth faces dramatic environmental changes caused by the living habits of an increasing human population ${ }^{1-3}$ which has consequences on life quality. A combination of effects from man-made global climate change, land degradation and contamination may compromise food production, for instance. Although traditional breeding and biotechnology are likely to overcome part of these constraints by engineering plants matching the environment ${ }^{4}$, more ecological and friendly practices would be highly beneficial as means to reduce the use of agro-pesticides and/or to enhance the environmental tolerance in agroecosystems. In this context, microbial symbionts of plants appear a promising alternative for improving plant performance and maintaining, or even increasing, the yield of crops $^{5-11}$

Soil salinization as a result of inappropriate cultural practices and excessive agricultural use is a symptom of land degradation. It currently affects a vast territory of productive areas throughout the world ${ }^{12-14}$. Additionally, agriculture expands to regions that are naturally affected by salinity. Therefore, plant resistance to salt, mainly to the sodium cation $\left(\mathrm{Na}^{+}\right)$, is a desirable trait in cultivated plants. One mechanism of plant tolerance in glycophytic (i.e. salt susceptible) species consists of reducing the cytoplasmic sodium concentration by sequestering $\mathrm{Na}^{+}$in the vacuole via tonoplast $\mathrm{Na}^{+} / \mathrm{H}^{+}$antiporters ${ }^{15,16}$. Apart from reducing sodium toxicity, increased ion

${ }^{1}$ Instituto de Ciencias Biológicas, Universidad de Talca, Campus Talca, Chile. ${ }^{2}$ Centro de Estudios Avanzados en Zonas Áridas (CEAZA), Universidad Católica del Norte, Coquimbo, Chile. ${ }^{3}$ Centro de Investigaciones y Estudios Avanzados del Maule (CIEAM), Universidad Católica del Maule, Talca, Chile. ${ }^{4}$ Grupo de Biodiversidad y Cambio Global (BCG), Departamento de Ciencias Básicas, Universidad del Bío-Bío, Chillán, Chile. ${ }^{5}$ IFEVA, Universidad de Buenos Aires, CONICET, Facultad de Agronomía, Buenos Aires, Argentina. ${ }^{6}$ Centro de Bioinformática y Simulación Molecular (CBSM), Facultad de Ingeniería, Universidad de Talca, Campus Talca, Chile. *email: marco.molina@utalca.cl 
concentrations in vacuoles allow plants to alleviate water deficit and maintain positive carbon gain ${ }^{15,17}$. The $\mathrm{Na}^{+}$ transport is governed by a family of genes of the NHX-type, well described in Arabidopsis thaliana but also present in other crop species ${ }^{7,10,11,18}$. Tomato plants are able to accumulate $\mathrm{Na}^{+}$in leaves when growing in soils with high sodium contents ${ }^{19}$ and an increased tolerance has been observed in plants overexpressing NHX genes ${ }^{20,21}$. It has also been scrutinized how plant tolerance to sodium can be improved by the association with root microorganisms ${ }^{22,23}$. For example, inoculation with arbuscular mycorrhizal fungi (AMF) significantly improved yield in tomato under high salt conditions ${ }^{24,25}$. In this case, the fungus supports the exclusion of $\mathrm{Na}^{+}$from the plants. In both, saline and no-saline soils, AMF-colonized plants had a lower $\mathrm{Na}^{+}$concentration than in non-colonized plants ${ }^{26}$. Root symbiotic microorganisms seem to activate the immune system of plants and increase overall performance, even in saline soils. It is unknown, however, in which way the presence of (a) root symbiont(s) cause(s) reduced $\mathrm{Na}^{+}$toxicity.

The association of plants with multiple microorganisms (e.g., fungal endophytes) either in roots or aboveground tissues is not the exception but the rule ${ }^{26-29}$. Some of the often cited effects of fungal endophytes are the gain of new metabolic capabilities by the host, the provision with secondary metabolites, and the stimulation of plant defense systems against abiotic stress factors and enemies ${ }^{8,30-33}$. For example, tomato plants (Solanum lycopersicum) that were grown on native soils showed an elicited immune response related to protection against oxidative stress compared to conspecific plants grown on sterile soils ${ }^{34}$. This effect was partially reverted when the plants growing in sterile soils were inoculated with the AMF Funneliformis mossea $e^{34}$. Arbuscular mycorrhizal fungi also improved the general performance of Robinia pseudoacacia seedlings under salt stress ${ }^{35}$. The overall physiological status of the plants (photosynthetic rate, photosystem II quantum efficiency and relative water content) was boosted by the symbiont, and genes encoding membrane transport proteins involved in $\mathrm{K}^{+} / \mathrm{Na}^{+}$ homeostasis in roots were upregulated ${ }^{35}$. Although AM fungi are by far the most studied soil microorganisms ${ }^{33-36}$, other root colonizers (e.g., Trichoderma spp., Piriformospora indica, Penicillium spp.) are known to deliver benefits to their host plants, too. Strains of Trichoderma, a common soil fungus, increased the tolerance to $\mathrm{Na}^{+}$in Arabidopsis thaliana by increasing the level of the auxin IAA (indole-3-acetic acid), the production of osmolites and antioxidants ${ }^{37}$.

Symbiotic fungi are proposed to be beneficial for the adaptation of plants to environmental stress factors $^{33,38-40}$. Different bio-prospective research programs started to consider fungi present in harsh environments as an unexplored source to find new metabolic pathways and ultimately, new bioactive constituents ${ }^{41}$. In this context, Antarctica has truly unique ecosystems representing one of the most severe climatic conditions for life on earth; i.e., low water availability, high UV-B radiation, extreme low temperatures and saline soils ${ }^{42}$. Nevertheless, there are plants and microorganisms that inhabit this harsh environment. Interestingly, in some cases these microorganisms have been found already to improve plant adaptation to stressful conditions by establishing a so-called 'functional symbiosis' ${ }^{\text {'3 }}$. Indeed, using the Antarctic plant Colobanthus quitensis as a source, we recently identified two species of Penicillium that retained their functional role observed in their native plant host even when exposed to a new plant species ${ }^{7,11}$. Thus, considering the geographic isolation and inhospitable conditions for growth that prevails in Antarctica and the referred ecological role of some Antarctic plant endophytes, it is highly plausible that these microorganisms may have unique metabolic pathways with an unexplored potential as biotechnological tools. Antarctic endophytes are thus an interesting new source for strategies using microbial plant symbionts for the adaptation of crops to the large environmental alterations predicted for the ongoing climate change ${ }^{44}$.

In this article, we report the beneficial effect of fungal endophytes isolated from roots of plants native to the Antarctic continent on the individual performance and final yield of two crop species, Lactuca sativa and Solanum lycopersicum, under salt stress. In order to get an insight into the underlying mechanisms, we assessed whether the endophyte-mediated improvement of host plant performance under sodium stress is related with enhanced individual physiological processes such as photosynthesis and water use efficiency, as well as with an upregulation of vacuolar $\mathrm{NHX} \mathrm{H}^{+} / \mathrm{Na}^{+}$antiporters. Finally, we demonstrated that the improved energy production goes hand in hand with an improved pathway for vacuolar $\mathrm{Na}^{+}$sequestration, suggesting an astonishing engineering capacity by Antarctic endophytes.

\section{Methodology}

Inoculation by fungal endophytes. We used a mix of two Antarctic fungal endophytes (AFE) isolated from the roots of two Antarctic plants, Colobanthus quitensis (AFE001) and Deschampsia antarctica (AFE002), during the growing season 2016-2017. Details concerning the procedures of fungal isolation, molecular characterization and species identification of these two fungal endophytes can be found in a previous study ${ }^{7}$. The isolates AFE001 (Genebank accession number: KJ881370) and AFE002 (Genebank accession number: KJ881371) were identified as Penicillium brevicompactum and P. chrysogenum, respectively ${ }^{7}$. These inoculums are maintained as part of the collection of microorganisms in the Plant Ecology Laboratory, Universidad de Talca, Chile. The inoculums were separated in different Petri dishes and then frozen until they were used for the experiments. We used these inoculums in combination because they are the most abundant endophytes found in the two Antarctic vascular plant species.

Fresh inoculums were obtained in March 2017 from single-conidia of AFE001 and AFE002 cultured on potato dextrose agar (PDA) medium diluted eight times and supplemented with $50 \mathrm{mg} / \mathrm{ml}$ of streptomycin. Cultures with endophytes were incubated at $22 \pm 2{ }^{\circ} \mathrm{C}$ and $350 \mu \mathrm{mol} \mathrm{m}^{-2} \mathrm{~s}^{-1}$ with a photoperiod $14 / 10$ day/night. After two weeks of incubation, conidia were harvested from plates by adding $10 \mathrm{ml}$ of sterile water and gently scraping off conidia with a sterile glass slide. The conidia suspension was adjusted to $100 \mathrm{ml}$ of $0.05 \%$ Tween-100, sterilized solution, filtered through three layers of sterile cotton cheesecloth gauze. Conidia concentration was estimated by using a Neubauer chamber and adjusted to $1 \times 10^{5}$ conidia $/ \mathrm{ml}$ and its viability was tested according to the methodology described by Greenfield et al.$^{45}$; the mean conidia viability was $>95 \%$. 
The endophyte inoculum consisted of a concentrated mix of conidia from the two fungi (P. brevicompactum and $P$. chrysogenum). Plants were inoculated with a $\sim 2 \mathrm{ml}$ suspension containing a concentrated mix of spores $\left(5000\right.$ spores $\mathrm{ml}^{-1}$ ) from each fungal endophyte directly applied to the rhizosphere of each individual plant. This procedure was repeated twice ( 15 days apart) to ensure the fungi to establish an effective association; verification of the symbiosis was evidenced by microscopy (Motic BA 310, with camera Moticam 2500) using root smears re-cultured on plates. Before the beginning of the experiment, two plants of each species/treatment were sacrificed to check microscopically for the presence or absence of endophytes by using a dissection microscope (Motic BA410, Chinese Group, CO., Ltda.) after cleaning the roots in $10 \% \mathrm{KOH}(\mathrm{w} / \mathrm{v})$ and staining with trypan blue in an acid glycerol solution.

Host plant species and experimental design. Lactuca sativa L. (lettuce var. Romaine) and Solanum lycopersicum L. (tomato var. Moneymaker) seedlings were obtained from seeds germinated in the glasshouse located at the Universidad de Talca, Talca, Chile $\left(35^{\circ} 24^{\prime} \mathrm{S} ; 71^{\circ} 37^{\prime} \mathrm{W}\right)$, under semi-controlled environmental conditions of light and temperature $\left(760 \pm 96 \mu \mathrm{mol} \mathrm{m}{ }^{-2} \mathrm{~s}^{-1}\right.$ with a photoperiod of $14 / 10 \mathrm{day} / \mathrm{night}$, and $\left.22 \pm 5^{\circ} \mathrm{C}\right)$. For treatment setup, lettuce and tomato seedlings previously inoculated in the laboratory were transplanted into the field in Chilean springtime of 2018 when individuals presented at least four expanded leaves and 3-cm roots. One-hundred seedlings of each species were randomly assigned to one of the four treatments: $(i)$ plants without the endophytes were irrigated with $40 \mathrm{~mL}$ of tap water plus $50 \mathrm{mM} \mathrm{NaCl}$ per day (Soil Electrical Conductivity: $6.8 \pm 1.7 \mathrm{mS} \mathrm{cm}^{-1}$ ), (ii) plants with the endophytes were irrigated with $40 \mathrm{~mL}$ of tap water plus $50 \mathrm{mM} \mathrm{NaCl}$ per day (SEC: $7.5 \pm 1.9 \mathrm{mS} \mathrm{cm}^{-1}$ ), (iii) plants without the endophytes were irrigated with $40 \mathrm{~mL}$ of tap water plus $150 \mathrm{mM} \mathrm{NaCl}$ per day (SEC: $17.6 \pm 1.2 \mathrm{mS} \mathrm{cm}^{-1}$ ), and (iv) plants with the endophytes were irrigated with $40 \mathrm{~mL}$ of tap water plus $150 \mathrm{mM} \mathrm{NaCl}$ per day (SEC: $17.2 \pm 1.5 \mathrm{mS} \mathrm{cm}^{-1}$ ). The irrigation of the plants remained constant during the experiment.

The amount of tap water that is normally added to lettuce and tomato crops in different commercial stations in the Maule region of Chile for this time of the year, ranges from 30 to $45 \mathrm{~mL} /$ day per plant. The seedlings were transplanted to the field and distributed in rows with a distance between plants of $0.2 \mathrm{~m}$ and a distance between rows of $0.5 \mathrm{~m}$. Each treatment was assigned to independent rows with four rows per each treatment. The soil of the plot is characterized by high content of clay, good drainage, and low levels of salt $(49 \pm 5 \mathrm{mM} \mathrm{NaCl}$; unpublished data). Each individual was fertilized with $0.2 \mathrm{~g} \mathrm{~L}^{-1}$ of Phostrogen (Solaris, NPK, 14:10:27) every 30 days. The experiment lasted for 90 (lettuce) and 100 (tomato) days, and the measurements were carried out simultaneously in all treatments for both species. Environmental conditions were recorded at midday (12:00-15:00 h) during the whole experimental period. Air temperature and relative humidity was recorded with a data logger (HOBO-Pro v2 U-23) and sunlight was registered with a portable photosynthetic active radiation sensor (Li-190 quantum sensor). During the experiment, daily mean temperature and relative humidity were $21.6^{\circ} \mathrm{C}( \pm 3.8)$ and $65 \%( \pm 12)$, respectively; while daily mean radiation was $1,422 \mu \mathrm{mol} \mathrm{m}^{-2} \mathrm{~s}^{-1}( \pm 336)$.

Crop yield. For each treatment, individuals of lettuce and tomato arranged were extracted from the soil without damaging the root system at the end of the experimental period. Then, the roots were washed carefully without removing them from the stem and left to dry in the shade for $1 \mathrm{~h}$. Total fresh biomass of both, shoots and roots of each individual was determined with a digital electronic scale (Boeco BBL-52; 0.01 g-precision). Taking into account the nature of the commercialized product for each species, the individual average final crop yield was estimated after over-drying at $62^{\circ} \mathrm{C}$ for $96 \mathrm{~h}$ the complete shoot tissues of each lettuce (i.e. the leaves), and the fruits in the case of tomato plants.

Plant survival. A total of 200 plants of lettuce and 200 plants of tomato were used for this experiment, where half of them $(n=100)$ were randomly assigned to one of the four treatments described previously as well as to field spatial arrangement. Survival was recorded weekly for each individual in the field for 12 weeks.

Ecophysiological traits. We assessed plant ecophysiological performance by determining the net photosynthesis rate $(\mathrm{A})$ and transpiration rate $(\mathrm{E})$. These parameters were measured on visually healthy leafs using an infrared gas analyzer (IRGA, Infra-Red Gas Analyzer, CIRAS-2, PP-Systems Haverhill, USA). Additionally, we calculated the water use efficiency (WUE) as the ratio between photosynthetic rate and transpiration (A/E). This parameter has been shown to be a good indicator of plant water stress under contrasting microhabitat conditions since reductions in water availability are paralleled with increases in $\mathrm{WUE}^{46}$. The experimental design consisted of 25 individuals for each treatment from a full factorial design with repeated measurements. The three response variables (A, E and WUE) were recorded for the same individual at days 30, 60 and 90 (lettuce) or 100 (tomato).

Gene expression. Total RNA was extracted from leaves of 5 individuals per species/treatment at 0,30 and 90 (lettuce) or 100 (tomato) days old plants according to the protocol described elsewhere ${ }^{47}$. The yield and purity of the extracted RNA was checked using UV absorption spectra, whereas its integrity was visually determined by electrophoresis on agarose gels. DNA was removed from RNA samples by using TURBO DNA-free (Applied Biosystems, California, USA). The first strand cDNA was synthesized according to the method described elsewhere $^{48}$. Quantitative PCR (qPCR) was performed according to the manufacturer's instructions in a final volume of $20 \mu \mathrm{l}$ containing $12.5 \mu \mathrm{l}$ of the Fast SYBR Green PCR master mix (Applied Biosystems), 5 pmol of each primer and the cDNA. The Elongation Factor $1 a(E F 1 a)$ was used as reference gene to quantify the changes in the relative gene expression (up- or down-regulation) of $N H X 1$ genes. The following primers were used to amplify the EF1a: 5'-GTACGCATGGGTGCTTGACAAACTC-3' (forward) and 5'-ATCAGCCTGGGAGGTACCAGTAAT-3' (reverse). LsNHX1 amplicons ( 200 bp) were obtained using the following primers: $5^{\prime}$ - GACAGTCCTGGAAAAT CT-3' (forward) and 5'-TGTGCCCTGACCTCGTAAACTGAT-3' (reverse). LeNHX amplicons ( 200 bp) were obtained using the following primers: $5^{\prime}$-GCACTTCTGTTGCTGTGAGTTCCA-3' (forward) and 
5'- GGTTATCAGCCCAAACACC-3' (reverse).The conditions of PCR amplification were: an initial cycle of $30 \mathrm{~min}$ at $45^{\circ} \mathrm{C}$ and $2 \mathrm{~min}$ at $95^{\circ} \mathrm{C}$, followed by 40 cycles of $30 \mathrm{~s}$ at $95^{\circ} \mathrm{C}, 30 \mathrm{~s}$ at $60^{\circ} \mathrm{C}$ and $2 \mathrm{~min}$ at $72^{\circ} \mathrm{C}$, followed by a final step of $10 \mathrm{~min}$ at $72^{\circ} \mathrm{C}$. Cycle threshold $\left(\mathrm{C}_{\mathrm{T}}\right)$ values were determined according to the $2^{\Delta \Delta \mathrm{CT}} \operatorname{method}^{49}$. From the qRT-PCR efficiencies and the crossing point deviation, we estimated the relative expression and fold changes (FC) of the target gene (salt treatments vs. controls), by comparing the target gene and the reference gene ( $\log _{2}$ transformed) according to the method described elsewhere ${ }^{50}$.

Nutrient content. At the end of the experiment, the concentrations of elements ( $\mathrm{N}, \mathrm{P}, \mathrm{K}$ and $\mathrm{Na}$ ) and molecules $\left(\mathrm{NO}_{3}{ }^{-}\right.$and $\left.\mathrm{NH}_{4}{ }^{+}\right)$in the plant biomass were determined for seven individuals from each treatment and expressed as percentage on dry weight basis. All analyses were conducted in the Laboratory of Nutrient Analysis at the Universidad de Talca, Chile. Shoot nutrient concentrations were determined after dry-ashing (except for nitrogen). $\mathrm{NO}_{3}{ }^{-}$and $\mathrm{NH}_{4}{ }^{+}$were determined after $\mathrm{KCl}$ extraction; $\mathrm{P}$ by Bray- 1 method; $\mathrm{K}$, and $\mathrm{Na}$ after ammonium acetate extraction. $\mathrm{N}$ was determined via combustion analysis (CNS-2000 Macro Analyzer, Leco Inc., MI, USA). P, K and Na were measured by ICP-OES (Perkin Elmer Optima 3000DV, Wellesley, MA, USA).

Statistical analysis. Six response variables related with plant fitness, physiological performance, gene expression and nutritional status were analyzed to describe the role of the endophytes on the biological performance of lettuce and tomato under saline stress. A standard two-way analysis of variance (ANOVA) was used to evaluate the effect of saline stress and endophyte inoculation on the final yield and final $\mathrm{Na}^{+}$foliar content. To evaluate the effect of saline stress and endophyte inoculation on those variables measured along time (photosynthesis, water use efficiency (WUE) and gene expression), we used two-way repeated measures (rm) ANOVAs. Model fitting was performed with the aov function from the base R options using the individual nested in time as the random error structure which is allowed for homoscedastic and orthogonal designs. Shoot nutrient concentrations ( $\mathrm{N}, \mathrm{P}$ and $\mathrm{K}$ ) under the four experimental treatments, control/E-, control/E +, salt added/ $\mathrm{E}-$, and salt added/E+, were compared for lettuce and tomato using a one-way ANOVA. For the one-way and two-way standard ANOVAs, a posteriori differences between treatments were evaluated using Honest Significant Differences (HSD) Tukey tests. Normality and homogeneity of variance were assessed with Shapiro-Wilks and Barlett tests, respectively ${ }^{51}$. A posteriori comparison for the rmANOVA models between experimental groups was performed by the comparison of their Estimated Marginal Means (EMMs) by factor levels as supported by the function pairs in the emmeans R-package ${ }^{52}$. The Kaplan-Meier survival functions were derived from the censored data of each experimental group using the survfit function from the survival R-package ${ }^{53}$. To determine the effect of the experimental factors (i.e.: endophyte infection and saline stress) on the survival probabilities, a Cox proportional-hazard model analysis was performed for each species data using the coxph function of the same package. Further pairwise comparisons were performed using the Peto and Peto modification of the Gehan-Wilcoxon test as allowed in the pairwise_survdiff function, implemented in the survminer R-package ${ }^{54}$. The assumption of proportionality between experimental factors for proportional hazard models was verified with the cox.zph R-function ${ }^{53}$.

\section{Results}

To evaluate the stress-mitigating potential of a combination of two Antarctic fungal endophytes, Penicillium brevicompactum and $P$. chrysogenum, they were tested under salt-stress conditions on two crop species, lettuce (Lactuca sativa) and tomato (Solanum lycopersicum). As in normal agricultural growth conditions plants were irrigated daily with $40 \mathrm{ml}$ of water containing $50 \mathrm{mM}$ (control) and $150 \mathrm{mM} \mathrm{NaCl}$ (stress), respectively. This type of experiment was set up with plants inoculated with the two fungi and in the absence of the endophytes. The differently treated plants were compared with respect to crop yield, the leaf nutrient content, water use efficiency, and the net photosynthetic rate.

Crop yield. As expected, salt stress reduced the final yield of both crops drastically (Fig. 1, 50/-E vs. 150/-E, $-38 \%$ for lettuce; $-48 \%$ for tomato). This inhibitory effect, however, was strongly mitigated by endophyte inoculation (Fig. 1, 50/-E vs. 150/+E, $-13 \%$ for both crops). While the presence of the endophytes increased the yield in both control and stress conditions, their impact on final yield was stronger under saline stress. The endophytes provoked a yield increase of $+42 \%$ in lettuce and of $+68 \%$ in tomato under salt stress $(150 /-\mathrm{E}$ vs. $150 /+\mathrm{E})$, while in control conditions (50/-E vs. 50/ $+\mathrm{E}$ ) a rise of $+7 \%$ (lettuce) and $+11 \%$ (tomato) was observed, only (Fig. 1).

Plant survival. Besides the yield increase of the surviving plants also the survival probability improved due to the presence of the endophytes under salt stress. To pinpoint this effect, we described the survival rates in our experiments (Fig. 2) with a Cox proportional hazards model (Table 1). The resulting hazard ratio (HR) described changes in the mortality risk due to the different treatments; $\mathrm{HR}=1$ means that the treatment did not affect mortality risk, $\mathrm{HR}>1$ means mortality risk has increased, $\mathrm{HR}<1$ means that mortality risk has decreased. The Cox regression estimated that the hazard ratios of the applied salt stress were $2.2 \pm 0.7$ (lettuce) and $3.3 \pm 1.1$ (tomato), respectively. This means that the salt stress significantly increased the mortality rates of the plants. When the salt stress was applied in combination with endophyte inoculation, however, the hazard ratios were not significantly different from 1 ( $1.2 \pm 0.6$ for lettuce and $1.7 \pm 1.0$ for tomato). Thus, the endophytes compensated for the salt stress resulting in an unchanged mortality rate. In low salt conditions, inoculation with the endophytes resulted in hazard ratios of $0.7 \pm 0.3$ (lettuce) and $0.4 \pm 0.2$ (tomato) indicating that the presence of the endophytes mitigated the mortality risk of both species even in control conditions.

Ecophysiological Traits. To get an idea on how the endophytes improved the fitness of the plants, we analyzed their photosynthetic capacity (net photosynthetic rate $\mathrm{A}_{\max }$; Fig. $3 \mathrm{~A}$ ) and estimated the water-use efficiency (WUE; Fig. 3B) for photosynthesis as the ratio between photosynthetic rate and transpiration. WUE is 


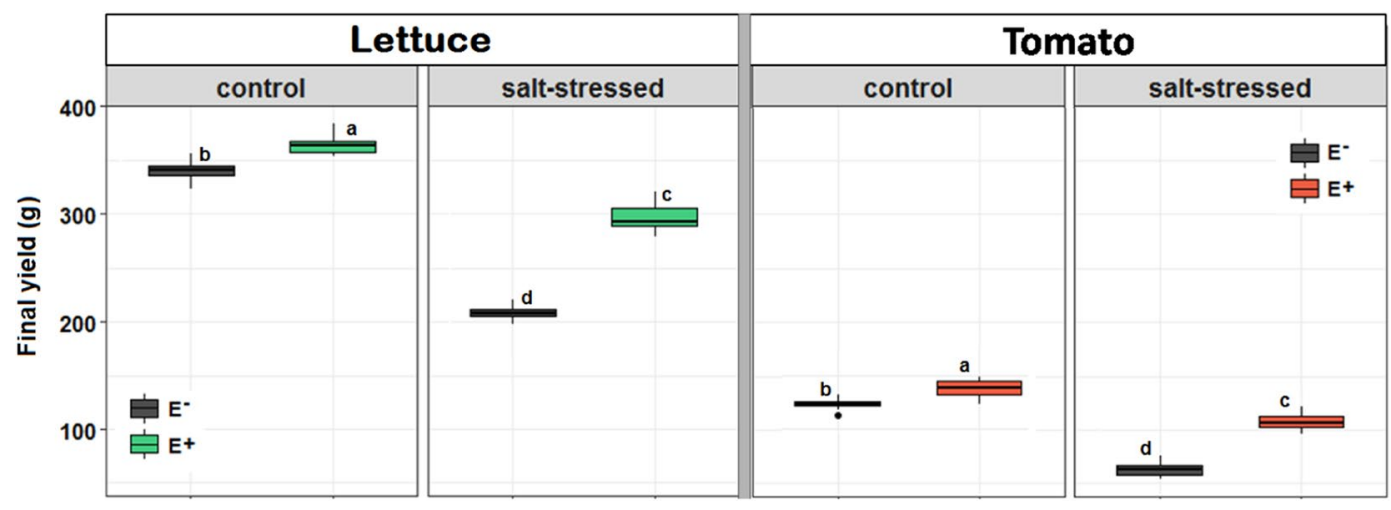

Figure 1. Effect of saline treatment ( $50 \mathrm{mM} \mathrm{NaCl}$, control, vs. $150 \mathrm{mM} \mathrm{NaCl}$, salt-stressed) and fungal inoculation (E - vs. E+) on the final yield of lettuce (left) and tomato (right) at the end of the experiment. Different lowercase letters indicate, for each species separately, significant differences between treatments (Tukey HSD tests; $\alpha=0.05)$. Values are means \pm SD $(n=25)$.
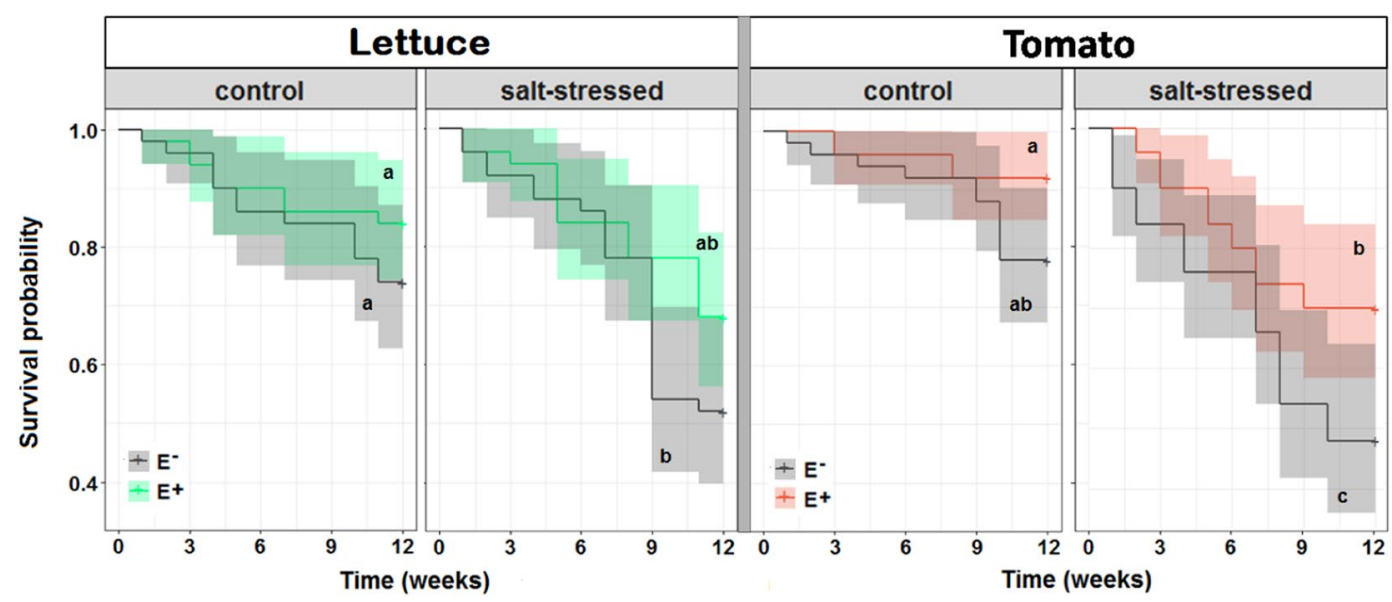

Figure 2. Temporal development of the survival rate of lettuce (left) and tomato plants (right) in the presence $(\mathrm{E}+)$ or absence $(\mathrm{E}-)$ of endophytes under salt stress $(150 \mathrm{mM} \mathrm{NaCl})$ and in control conditions $(50 \mathrm{mM} \mathrm{NaCl})$. For each condition, 50 plants were selected and their survival was monitored over time.

\begin{tabular}{|c|c|c|c|c|}
\hline \multirow[b]{2}{*}{ Factor } & \multicolumn{2}{|l|}{ Lettuce } & \multicolumn{2}{|l|}{ Tomato } \\
\hline & $\beta \pm \mathrm{SE}$ & $\operatorname{HR}\left(e^{\beta}\right)$ & $\beta \pm \mathrm{SE}$ & $\operatorname{HR}\left(e^{\beta}\right)$ \\
\hline $\mathrm{E}+$ & $-0.52 \pm 0.45$ & $0.7 \pm 0.3$ & $-1.04 \pm 0.58$ & $0.4 \pm 0.2$ \\
\hline salt stress & $0.75 \pm 0.34$ & $2.2 \pm 0.7$ & $1.13 \pm 0.36$ & $3.3 \pm 1.1$ \\
\hline $\mathrm{E}+/$ salt stress & $0.01 \pm 0.55$ & $1.2 \pm 0.6$ & $0.35 \pm 0.67$ & $1.7 \pm 1.0$ \\
\hline
\end{tabular}

Table 1. Parameters of the regressions of the Cox proportional-hazards models. For both species the three influencing factors (i) $\mathrm{E}+$ (with endophytes in $50 \mathrm{mM} \mathrm{NaCl}$ ), (ii) salt stress (without endophytes in $150 \mathrm{mM}$ $\mathrm{NaCl}$ ), and (iii) $\mathrm{E}+/$ salt stress (with endophytes in $150 \mathrm{mM} \mathrm{NaCl}$ ) were evaluated against the control condition (without endophytes in $50 \mathrm{mM} \mathrm{NaCl}$ ). The models are based on 200 individuals for each species monitored for 12 weeks and 61 lethal events for lettuce and 56 for tomato. HR: hazard ratio, SE: standar error. The variance of $\mathrm{HR}$ was calculated on the basis of the $95 \%$ confidence interval of $\beta\left([\beta-\mathrm{SE} . . \beta+\mathrm{SE}] \rightarrow\left[\mathrm{HR}_{\min } . . \mathrm{HR}_{\max }\right]\right)$.

an indicator of plant water stress in a microenvironment because an increase in WUE is usually induced by a decrease in water availability ${ }^{46}$.

In both crops without endophytes, salt stress reduced significantly $A_{\max }(-33 \%$ in lettuce and $-28 \%$ in tomato at the end of the cultivation period) and WUE ( $-22 \%$ in lettuce and $-31 \%$ in tomato) (Fig. 3B; Table S1). However, when the stress was applied to plants with endophytes, the negative effect of salt stress on the net photosynthetic rate was compensated ( $-4 \%$ in lettuce and $-1 \%$ in tomato) and the WUE was even improved $(+20 \%$ in lettuce and $+11 \%$ in tomato). The positive effect of the endophytes was more pronounced in stress conditions. While their inoculation resulted in an increase of $\mathrm{A}_{\max }$ of $+24 \%$ (lettuce) and $+19 \%$ (tomato) in 


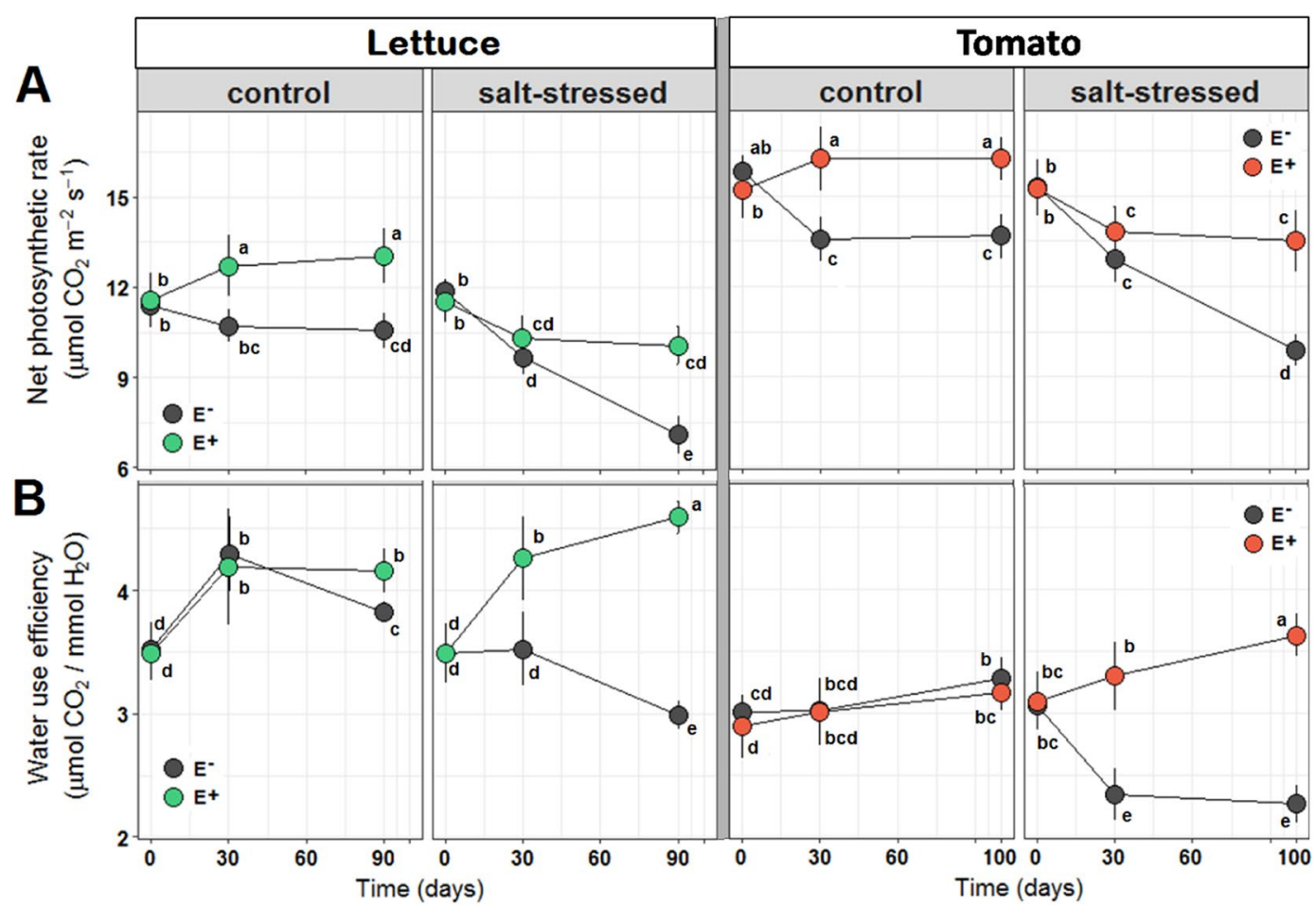

Figure 3. Effect of saline treatment ( $50 \mathrm{mM} \mathrm{NaCl}$, control, vs. $150 \mathrm{mM} \mathrm{NaCl}$, salt-stressed) and fungal inoculation (E- vs. E+) on the net photosynthetic rate $\left(A_{\text {max }} ; \mathrm{A}\right)$ and the water use efficiency (WUE; $\left.\mathrm{B}\right)$ of lettuce (left) and tomato (right). Different lowercase letters indicate, for each species separately, significant differences between treatments (Tukey HSD tests; $\alpha=0.05)$. Values are means \pm SD $(n=20)$.

\begin{tabular}{|l|l|l|l|l|l|}
\hline \multirow{3}{*}{ Species } & \multirow{3}{*}{ Control } & \multicolumn{2}{l|}{ Salt-stressed } \\
\cline { 3 - 6 } & Nutrient $(\%)$ & E + & E - & E + & E - \\
\hline \multirow{3}{*}{ Lettuce } & Nitrogen & $3.5 \mathrm{a}( \pm 0.16)$ & $3.1 \mathrm{~b}( \pm 0.13)$ & $2.8 \mathrm{c}( \pm 0.18)$ & $2.2 \mathrm{~d}( \pm 0.22)$ \\
\cline { 2 - 6 } & Phosphorus & $0.7 \mathrm{a}( \pm 0.11)$ & $0.6 \mathrm{a}( \pm 0.09)$ & $0.4 \mathrm{~b}( \pm 0.11)$ & $0.3 \mathrm{~b}( \pm 0.11)$ \\
\cline { 2 - 6 } & Potassium & $7.1 \mathrm{~b}( \pm 0.81)$ & $6.9 \mathrm{~b}( \pm 1.07)$ & $10.1 \mathrm{a}( \pm 0.54)$ & $8.2 \mathrm{~b}( \pm 0.89)$ \\
\hline \multirow{3}{*}{ Tomato } & Nitrogen & $6.1 \mathrm{a}( \pm 0.14)$ & $5.4 \mathrm{~b}( \pm 0.17)$ & $3.6 \mathrm{c}( \pm 0.21)$ & $2.9 \mathrm{~d}( \pm 0.19)$ \\
\cline { 2 - 6 } & Phosphorus & $0.8 \mathrm{a}( \pm 0.11)$ & $0.7 \mathrm{a}( \pm 0.12)$ & $0.3 \mathrm{~b}( \pm 0.11)$ & $0.3 \mathrm{~b}( \pm 0.09)$ \\
\cline { 2 - 6 } & Potassium & $9.5 \mathrm{~b}( \pm 0.33)$ & $7.8 \mathrm{c}( \pm 0.42)$ & $12.7 \mathrm{a}( \pm 0.39)$ & $11.5 \mathrm{a}( \pm 0.41)$ \\
\hline
\end{tabular}

Table 2. Concentration of nutrients (\% of dry weight) in foliar tissues of lettuce and tomato plants grown for 90 and 100 days, respectively, under $50 \mathrm{mM} \mathrm{NaCl}$ (Control) and $150 \mathrm{mM} \mathrm{NaCl}$ (Salt-stressed) in the presence $(\mathrm{E}+$ ) or absence $(E-)$ of root fungal endophytes. Values are means $\pm S D(n=20)$. Different lowercase letters indicate significant differences among treatments for each nutrient by species combination (Tukey HSD tests; $\alpha=0.05$ ).

control conditions, this value increased to $+43 \%$ (lettuce) and $+37 \%$ (tomato) in salt stress conditions. The same applies to the WUE, with only marginal variations in low salt, and a significant increase of $+54 \%$ (lettuce) $/+60 \%$ (tomato) in salt stress (Fig. 3B). The increase of the net photosynthetic rate in control conditions indicates that the endophytes alone induced already a partially latent stress-response in the crop plants. This initial response then largely compensated for the inhibitory effect of salt stress on the net photosynthetic rate and overcompensated for the detrimental effect on WUE (Fig. 3A,B). Thus, the presence of the endophytes improved plant fitness in salt stress conditions by mitigating the negative effects on an efficient energy production.

Nutrient content. As next, we analyzed in which way salt stress affected the content of the key nutrients nitrogen, phosphorus and potassium in the crops (Table 2). In the absence of endophytes, the application of salt stress resulted in an increase of the potassium content and a decrease of the nitrogen and phosphate content in both crops. The presence of endophytes mitigated the inhibitory effect on the nitrogen content and stimulated further the accumulation of potassium (in lettuce only, not in tomato), but had no significant influence on the phosphorus content. Even under low salt, the presence of the endophytes stimulated potassium (in tomato only, not in lettuce) and nitrogen accumulation (Table 2). The increased nitrogen and potassium content after endophyte inoculation pointed to an increased uptake of these elements from the soil. 


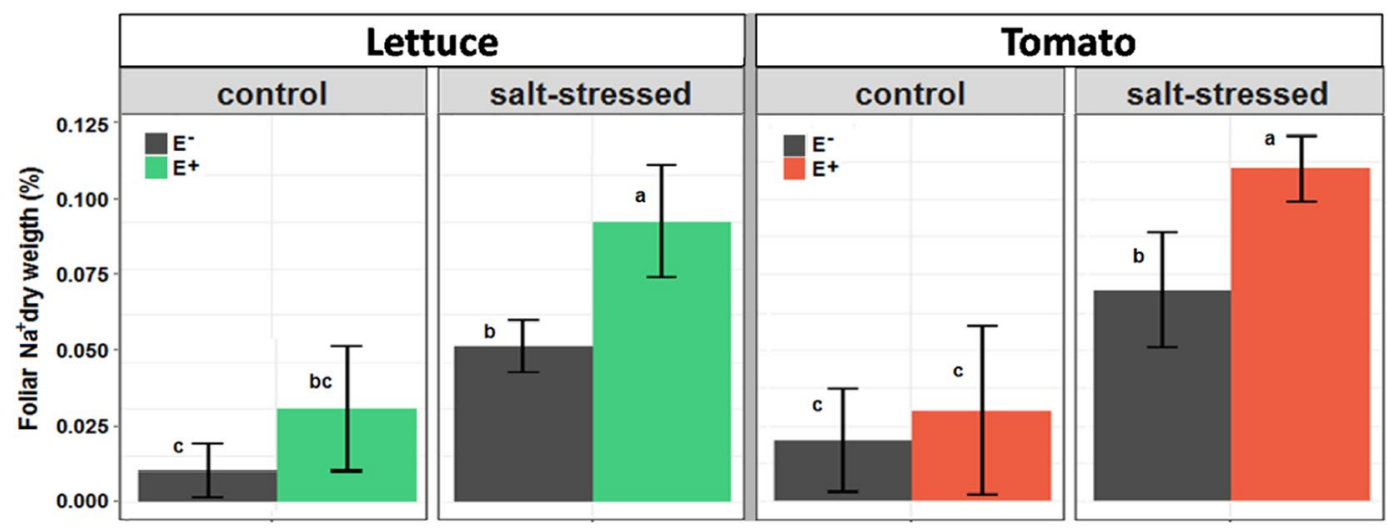

Figure 4. Foliar $\mathrm{Na}^{+}$content of lettuce (left) and tomato plants (right) grown for 90 (lettuce) or 100 days (tomato) under $50 \mathrm{mM} \mathrm{NaCl}$ (control) and $150 \mathrm{mM} \mathrm{NaCl}$ (salt-stressed) in the presence $(+\mathrm{E})$ or absence $(-\mathrm{E})$ of root fungal endophytes. Values are means \pm SE $(n=20)$. Different lowercase letters indicate, for each species separately, significant differences between treatments (Tukey HSD tests; $\alpha=0.05$ ).

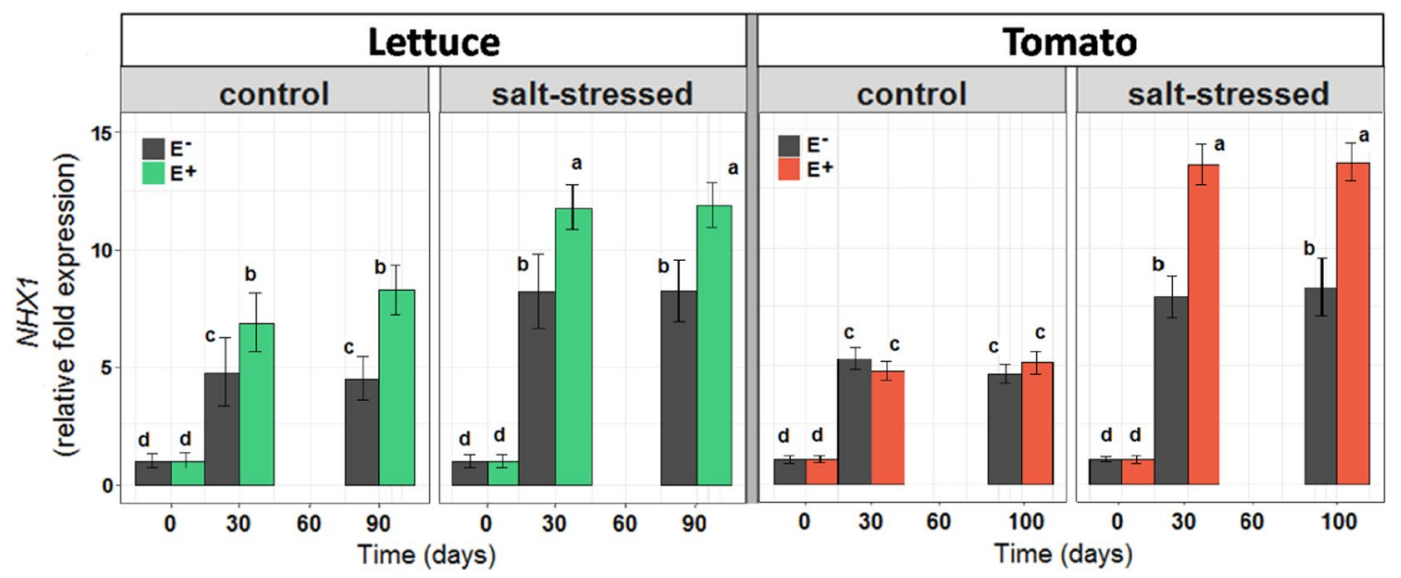

Figure 5. Effects of saline treatment (control vs. salt-stressed) and fungal inoculation (E- vs. E+ plants) on the expression level of the NHX1 gene of lettuce and tomato plants along time. Different lowercase letters indicate, for each species separately, significant differences between treatments (Tukey HSD tests; $\alpha=0.05$ ). Values are means \pm SE $(\mathrm{n}=20)$.

Content of foliar $\mathrm{Na}^{+}$and expression of vacuolar $\mathrm{NHX}$ exchanger. The exposure of uninoculated plants to salt stress resulted in a significant increase of the foliar sodium content in both crops (Fig. 4). However, this increment was even larger in endophyte-inoculated plants. In other words, the endophyte-specific effect was more pronounced under high salt condition. Under low salt concentration -please, notice that the control condition contains already a significant amount of $\mathrm{NaCl}$ (i.e. $50 \mathrm{mM}$ )-, the presence of endophytes provoked a slight increase in $\mathrm{Na}^{+}$content in lettuce but not in tomato. In contrast, under salt stress conditions the endophytes caused a larger accumulation of sodium in leaves (Fig. 4).

Elevated cytosolic sodium concentrations are toxic for plants. In our results, however, the elevated $\mathrm{Na}^{+}$content correlated with several improved physiological parameters. We therefore speculated that most of the sodium in the leaves has been sequestered in vacuoles, which is an efficient strategy to mitigate salt stress. One bottleneck for $\mathrm{Na}^{+}$-sequestration could be the availability of transport pathways into the vacuole. We therefore analyzed the expression level of the vacuolar $\mathrm{Na}^{+} / \mathrm{H}^{+}$antiporter $\mathrm{NHX} 1$. For both species L. sativa and S. lycopersicum we identified the sequences of the genes homologous to AtNHX1 and determined their expression levels by qRT-PCR in the four compared conditions (Fig. 5). It turned out that salt-stress alone already increased the NHX1 expression levels. This upregulation was boosted further by the presence of the endophytes. Under low salt conditions the endophytes stimulated $N H X 1$ expression in lettuce but not in tomato. All these results correlated very well with the levels of $\mathrm{Na}^{+}$ accumulation in leaves. A higher $\mathrm{NHX1}$ expression was accompanied by a higher foliar $\mathrm{Na}^{+}$content.

\section{Discussion}

The two fungal endophytes originally isolated from Antarctic plants, Penicillium chrysogenum and Penicillium brevicompactum, had previously been found responsible for increasing yield under water shortage and salinity conditions in lettuce ${ }^{7,11}$. Besides improving plant physiological parameters $\left(\mathrm{A}_{\max }\right.$ and WUE), fungal endophytes also promoted the accumulation of the amino acid proline ${ }^{7}$, an osmotic molecule that has been previously 
suggested to mediate symbiont-conferred plant tolerance to water deficit ${ }^{55,56}$. More recently, it was found that the root fungal endophyte Piriformospora indica improved the ecophysiological performance of tomato plants under salinity by regulating $\mathrm{Na}^{+} / \mathrm{K}^{+}$homeostasis and increasing the activity of antioxidant enzymes ${ }^{10}$. Antarctic fungal endophytes have been shown to be functional in the native Antarctic vascular plant Colobanthus quitensis ${ }^{43}$, but also when non-Antarctic plants were inoculated (e.g., Flourensia thurifera, Puya berteroniana and Senna cumingii ${ }^{57,58}$ ). Here we were able to find a relation between the improvements of individual plant performance and increased expression of the gene NHX1 involved in ionic homeostasis. The accumulating evidence suggests that these endophytes are generalist for establishing functional symbiosis with even non-natural hosts. This finding might provide the foundation for using these organisms as biological tool to improve crops in hard situations.

We tested the potential of the Antarctic root-fungal endophytes P. chrysogenum and P. brevicompactum to improve the tolerance of two cultivated species (i.e. lettuce and tomato) to high level of salt-stress. Here we observed that endophyte-inoculated plants produced more total biomass than non-inoculated counterparts. Although sodium caused a significant reduction in plant size, this negative effect was more pronounced on non-inoculated plants. For both host species, the survival probability over the experimental time, was higher for inoculated plants than for non-inoculated plants. Like the effect on biomass, the beneficial effect of the root fungal endophytes on this latter response variable was hierarchically more evident under high sodium in the soil. The context-dependence of symbiosis-outcomes are similar across plant species and microorganism types ${ }^{26}$. However, in order to predict the outcome of a symbiotic interaction under a given environmental context, it turns out to be critical to know the changes caused by the microorganism on the plant morphology and physiology. For example, root endophytes can induce changes in plant root architecture by deploying new roots toward less salty soils ${ }^{59,60}$. Although we did not assess morphological changes in the root system of the plants, the greater relative growth at the end of the experiments could be at least partly attributed to the positive effect of the endophytes on root functionality (e.g., higher water up-take). Improved membrane functionality, higher concentration of osmotically active molecules and increased antioxidant capacity produced or modulated by fungal endophytes could explain the ability of plants to grow even under stress conditions ${ }^{7,32}$.

Besides being a source of bioactive secondary metabolites, symbiotic microbial symbionts can reprogram gene expression of the host to adjust the plant phenotype for improved tolerance to abiotic stress factors ${ }^{32,61,62}$. Particularly under salt stress, the beneficial effects of the Antarctic fungal endophytes became most evident on plant growth and survival. The inoculation of roots with fungal endophytes changed the expression of the NHX1 gene in leaves of the two crop species. Although this gene may have different isoforms depending on the species ${ }^{63}$, it is involved in several functions related to the maintenance of the ionic balance and cell turgor ${ }^{10}$. High activity of NHX proteins $\left(\mathrm{Na}^{+} / \mathrm{H}^{+}\right.$antiporters integrated in vacuolar membranes) are associated with the capacity of accumulating $\mathrm{Na}^{+}$inside vacuoles and, in turn, with a higher tolerance of the plant to toxic sodic salt soils ${ }^{64}$. We observed that in high-sodium soil, endophyte-inoculated plants were able to accumulate higher concentrations of $\mathrm{Na}^{+}$in leaves compared to non-inoculated plants. The correlation of the up regulation of the vacuolar $\mathrm{Na}^{+} / \mathrm{H}^{+}$antiporter NHX1 with increasing soil salt levels and with increasing accumulation of $\mathrm{Na}^{+}$in leaves of endophyte-inoculated plants suggests a larger density of these transporters sequestering $\mathrm{Na}^{+}$in vacuoles ${ }^{65}$. This endophyte mediated mechanism would allow plants to maintain ionic homeostasis and capacity to grow under high levels of sodium in the soil. As we only measured the expression associated to one of the NHX possible isoforms ${ }^{63}$, we can not specify whether this endophyte-mediated effect is general on all the isoforms or whether it is specific for NHX1. However, in the context of other publications reporting similar patterns and results (see e.g. $\left.{ }^{10,66}\right)$, our findings suggest that the effects of root fungal endophytes on plant tolerance to salt are general.

Central to any biotechnological breeding strategy on crop plants is that any change at the molecular level translates into improved physiological activity and ultimately, impacts on plant biomass accumulation. Here we observed that endophyte inoculated plants that showed an increased expression of the NHX1 gene, displayed also higher photosynthetic capacity and water used efficiency. Compared to non-inoculated plants, the positive effects of the inoculated root endophytes on plant photosynthesis and WUE were more apparent under salinity conditions and as plants became bigger. A higher expression of NHX1 might be necessary but is not sufficient for a higher vacuolar $\mathrm{Na}^{+}$transport rate, as such transport consumes energy ${ }^{67,68}$. Therefore, a positive feedback between different processes could explain the overall better performance of plants under stress. The improved photosynthetic rates and reduced transpiration rates of endophyte-inoculated plants, would be essential for maintaining successful sequestration of $\mathrm{Na}^{+}$. A reduced transpiration rate decreases the passive influx of $\mathrm{Na}^{+}$ions into the roots ${ }^{69}$ and thus minimizes the energy required for $\mathrm{Na}^{+}$extrusion and/or sequestration. An improved photosynthesis rate can contribute to cover the increased energy demand for driving the $\mathrm{H}^{+}$-ATPases and PPases that need to build up and maintain the electrical and proton gradients for an efficient $\mathrm{Na}^{+} / \mathrm{H}^{+}$antiport for $\mathrm{Na}^{+}$sequestration. Thus, lettuce and tomato plants were stimulated by the Antarctic root endophytes to orchestrate a diverse set of physiological parameters in a concerted action which finally allowed them to cope successfully with a salt stress condition. We can, therefore, conclude that the two endophytes Penicillium brevicompactum and P. chrysogenum mitigated salt stress in lettuce and tomato, provoking an efficient gain of carbon through a higher photosynthetic energy production and controlling the negative effect of sodium ions, likely by sequestration in vacuoles.

The growing demand for goods, overexploitation of agricultural lands and global climate change have promoted a rapid expansion of saline soils worldwide, threatening regular practices of agriculture and food security ${ }^{44}$. Under such scenarios, the physiological and biochemical functions of both wild and cultivated plant species are impaired causing severe yield losses ${ }^{59}$. Although it still needs to be tested at real production scales, harnessing fungal endophyte symbionts of plants appears to be a suitable strategy not only for improving crop yields but also for the design of environmentally friendly agricultural strategies ${ }^{5,6,11,14}$.

Received: 30 October 2019; Accepted: 17 February 2020;

Published online: 02 April 2020 


\section{References}

1. Tilman., D. et al. Agricultural sustainability and intensive production practices. Nature 418, 671-677 (2002).

2. Foley, J. A. et al. Global consequences of land use. Science 309, 570-574 (2005).

3. Godfray, H. C. et al. Food security: the challenge of feeding 9 billion people. Science 327, 812-818 (2010).

4. Carroll, S. P. et al. Applying evolutionary biology to address global challenges. Science 346, 313-323 (2014).

5. Gundel, P. E., Pérez, L. I., Helander, M. \& Saikkonen, K. Symbiotically modified organisms: non-toxic fungal endophytes in grasses. Trends Plant Sci. 18, 420-427 (2013).

6. Kauppinen, M., Saikkonen, K., Helander, M., Pirttilä, A. M. \& Wäli, P. R. Epichloë grass endophytes in sustainable agriculture. Nat. Plants 2, 1-7 (2016).

7. Molina-Montenegro, M. A. et al. Root-endophytes improve the ecophysiological performance and Production of an Agricultural Species under Drought Condition. AoB Plants 8, plw062 (2016a).

8. Hawkes, C. V. \& Connor, E. W. Translating phytobiomes from theory to practice: ecological and evolutionary considerations. Phytobiomes J. 1, 57-69 (2017).

9. Wei, Z. \& Jousset, A. Plant breeding goes microbial. Trends Plant Sci. 22, 555-558 (2017).

10. Abdelaziz, M. E. et al. Piriformospora indica alters $\mathrm{Na}^{+} / \mathrm{K}^{+}$homeostasis, antioxidant enzymes and LeNHX1 expression of greenhouse tomato grown under salt stress. Sci. Hortic. 256, 108532 (2019).

11. Acuña-Rodríguez, I. S., Hansen, H., Gallardo-Cerda, J., Atala, C. \& Molina-Montenegro, M. A. Antarctic extremophiles: biotechnological alternative to crop productivity in saline soils. Front. Bioeng. Biotechnol. 7, 22 (2019).

12. Metternicht, G. I. \& Zinck, J. A. Remote sensing of soil salinity: potentials and constraints. Remote Sens. Environ. 85, 1-20 (2003).

13. Qadir, M. et al. Economics of salt-induced land degradation and restoration. Nat. Resour. Forum 28, 282-295 (2014).

14. Roy, S. J., Negraro, S. \& Tster, M. Salt resistant crop plants. Curr. Opin. Biotech. 26, 115-124 (2014).

15. Blumwald, E., Aharon, G. S. \& Apse, M. P. Sodium transport in plant cells. Biochim. Biophys. Acta 1465, 140-151 (2000).

16. Munns, R. \& Tster, M. Mechanisms of Salinity Tolerance. Annu. Rev. Plant Biol. 59, 651-681 (2008).

17. Pardo, J. M. \& Quintero, F. J. Plants and sodium ions: keeping company with the enemy. Genome Biol. 3, 1017.1-1017.4 (2002).

18. Pardo, J. M., Cubero, B., Leidi, E. O. \& Quintero, F. J. Alkali cation exchangers: roles in cellular homeostasis and stress tolerance. J. Exp. Bot. 57, 1181-1199 (2006)

19. Zhang, H. X. \& Blumwald, E. Transgenic salt-tolerant tomato plants accumulate salt in foliage but not in fruit. Nat. Biotechnol. 19, 765-768 (2001).

20. Gouiaa, S. \& Khoudi, H. Co-expression of vacuolar $\mathrm{Na}(+) / \mathrm{H}(+)$ antiporter and $\mathrm{H}(+)$-pyrophosphatase with an IRES-mediated dicistronic vector improves salinity tolerance and enhances potassium biofortification of tomato. Phytochemistry 117, 537-546 (2015).

21. Leidi, E. O. et al. The AtNHX1 exchanger mediates potassium compartmentation in vacuoles of transgenic tomato. Plant J. 61, 495-506 (2010).

22. Hanin, M., Ebel, C., Ngom, M., Laplaze, L. \& Masmoudi, K. New insights on plant salt tolerance mechanisms and their potential use for breeding. Front. Plant Sci. 7, 1787 (2016).

23. Rho, H. et al. Do endophytes promote growth of host plants under stress? A meta-analysis on plant stress mitigation by endophytes. Microbial Ecol. 75, 407-418 (2017).

24. Al-Karaki, G. N. Growth, water use efficiency, and sodium and potassium acquisition by tomato cultivars grown under salt stress. J. Plant Nutr. 23, 1-8 (2000)

25. Latef, A. H. A. \& Chaoxing, H. Effect of arbuscular mycorrhizal fungi on growth, mineral nutrition, antioxidant enzymes activity and fruit yield of tomato grown under salinity stress. Sci. Hortic. 127, 228-233 (2011).

26. Rodriguez, R., White, J., Arnold, A. \& Redman, R. Fungal endophytes: diversity and functional roles. New Phytol. 182, 314-330 (2009).

27. Hardoim, P. R. et al. The hidden world within plants: ecological and evolutionary considerations for defining functioning of microbial endophytes. Microbiol. Mol. Biol. Rev. 79, 293-320 (2015).

28. Jin, K., White, P. J., Whalley, W. R., Shen, J. \& Shi, L. Shaping an optimal soil by root-soil interaction. Trends Plant Sci. 22, 823-829 (2017).

29. Lemanceau, P., Blouin, M., Muller, D. \& Moënne-Loccoz, Y. Let the core microbiota be functional. Trends Plant Sci. 22, 583-595 (2017).

30. Rodriguez, R. \& Redman, R. Balancing the generation and elimination of reactive oxygen species. P. Nat. Acad. Sci. USA 102, 3175-3176 (2005).

31. Pineda, A., Zheng, S. J., van Loon, J. J. A., Pieterse, C. M. J. \& Dicke, M. Helping plants to deal with insects: the role of beneficial soilborne microbes. Trends Plant Sci. 15, 507-514 (2010).

32. Hamilton, C. E., Gundel, P. E., Helander, M. \& Saikkonen, K. Endophytic mediation of reactive oxygen species and antioxidant activity in plants: a review. Fungal Divers. 54, 1-10 (2012).

33. Pieterse, C. M. J. et al. Induced systemic resistance by beneficial microbes. Annual Review of Phytopathol. 52, 347-375 (2014).

34. Chialva, M. et al. Native soils with their microbiotas elicit a state of alert in tomato plants. New Phytol. 220, 1296-1308 (2018)

35. Chen, J., Zhang, H., Zhang, X. \& Tang, M. Arbuscular mycorrhizal symbiosis alleviates salt stress in black locust through improved photosynthesis, water status, and $\mathrm{K}+/ \mathrm{Na}+$ homeostasis. Front. Plant Sci. 8, 1739 (2017).

36. Omacini, M., Semmartin, M. G., Perez, L. I. \& Gundel, P. E. Grass-endophyte symbiosis: a neglected aboveground interaction with multiple belowground consequences. Appl. Soil Ecol. 61, 273-279 (2012).

37. Contreras-Cornejo, H. A., Macías-Rodríguez, L., Alfaro-Cuevas, R. \& López-Bucio, J. Trichoderma spp. improve growth of Arabidopsis seedlings under salt stress through enhanced root development, osmolite production, and $\mathrm{Na}^{+}$elimination through root exudates. Mol. Plant Microbe In. 27, 503-514 (2014).

38. Clay, K. \& Schardl, C. Evolutionary origin and ecological consequences of endophyte symbiosis with grasses. Am. Nat. 160, S99-S127 (2002).

39. Redman, R. S., Sheehan, K. B., Stout, R. G., Rodriguez, R. J. \& Henson, J. M. Thermotolerance generated by plant/fungal symbiosis. Science 298, 1581 (2002).

40. Ramos, P., Rivas, N., Pollmann, S., Casati, P. \& Molina-Montenegro, M. A. Hormonal and physiological changes driven by fungal endophytes increase Antarctic plant performance under UV-B radiation. Fungal Ecol. 34, 76-82 (2018).

41. Godinho, V. M. et al. Diversity and bioprospecting of fungal communities associated with endemic and cold-adapted macroalgae in Antarctica. ISME Journal 7, 1434-1451 (2013).

42. Convey, P. et al. The spatial structure of Antarctic biodiversity. Ecol. Monogr. 84, 203-244 (2014).

43. Torres-Díaz, C. et al. Biological interactions and simulated climate change modulates the ecophysiological performance of Colobanthus quitensis in the Antarctic ecosystem. PLoS ONE 11, e0164844 (2016).

44. IPCC. (Intergovernmental Panel on Climate Change), www.ipcc.ch (2018)

45. Greenfield, M. et al. Beauveria bassiana and Metarhizium anisopliae endophytically colonize cassava roots following soil drench inoculation. Biol. Control 95, 40-48 (2016).

46. Lambers, H., Chapin, F. S. \& Pons, T. L. Plant Physiological Ecology. New York, Springer, Verlag, (1998).

47. Chang, S., Puryear, J. \& Cainey, J. A simple and efficient method for isolating RNA from pine trees. Plant Mol. Biol. Rep. 11, 113-116 (1993). 
48. Ruiz-Carrasco, K. et al. Variation in salinity tolerance of four lowland genotypes of quinoa (Chenopodium quinoa Willd.) as assessed by growth, physiological traits, and sodium transporter gene expression. Plant Physiol. Bioch. 49, 1333-1341 (2011).

49. Livak, K. J. \& Schmittgen, T. D. Analysis of relative gene expression data using real-time quantitative PCR and the $2 \Delta \Delta \mathrm{C}_{\mathrm{T}}$ method. Methods 25, 402-408 (2001).

50. Pfaffi, M. W. A new mathematical model for relative quantification in real-time RT-PCR. Nucleic Acid Res. 29, e45 (2001).

51. Sokal, R. R. \& Rohlf, F. J. Biometry: the principles and practice of statistics in biological research. New York: Freeman (1981).

52. Lenth, R. emmeans: Estimated Marginal Means, aka Least-Squares Means. R-package version 1.1.2., 2018, https://CRAN.R-project. org/package $=$ emmeans (2018)

53. Therneau, T. A Package for Survival Analysis in S v.2.38., 2015, https://CRAN.R-project.org/package=survival (2015).

54. Kassambara, A. \& Kosinski, M. survminer: Drawing Survival Curves using 'ggplot2'. R package version 0.4.2., 2018, http://www. sthda.com/english/rpkgs/survminer/ (2018).

55. Nagabhyru, P., Dinkins, R. D., Wood, C. L., Bacon, C. W. \& Schardl, C. L. Tall fescue endophyte effects on tolerance to water-deficit stress. BMC Plant Biol. 13, 127 (2013).

56. Ortiz, N., Armada, E., Duque, E., Roldán, A. \& Azcón, R. Contribution of arbuscular mycorrhizal fungi and/or bacteria to enhancing plant drought tolerance under natural soil conditions: effectiveness of autochthonous or allochthonous strains. J. Plant Physiol. 174, $87-96(2015)$.

57. Fardella, C., Oses, R., Torres-Díaz, C. \& Molina-Montenegro, M. A. Antarctic fungal endophytes as tool for the reintroduction of native plant species in arid zones. Bosque 35, 235-239 (2014).

58. Molina-Montenegro, M. A. et al. Nurse effect and soil microorganisms are key to improve the establishment of native plants in a semiarid community. J. Arid Environ. 126, 54-61 (2016b).

59. Deinlein, U. et al. Plant salt-tolerance mechanisms. Trends Plant Sci. 19,371-379 (2014).

60. Galván-Ampudia, C. S. \& Tasterink, C. Salt tress signals shape the plant root. Curr. Opin. Plant Biol. 14, 296-302 (2015).

61. Khan, A. L. et al. Fungal endophyte Penicillium janthinellum LK5 improves growth of ABA-deficient tomato under salinity. World J. Microb. Biot. 29, 2133-2144 (2013).

62. Harman, G. E., Howell, C. R., Viterbo, A., Chet, I. \& Lorito, M. Trichoderma species-opportunistic, avirulent plant symbionts. Nature Rev. Microbiol. 2, 43-56 (2004).

63. Gálvez, F. J. et al. Expression of LeNHX isoforms in response to salt stress in salt sensitive and salt tolerant tomato species. Plant Physiol. Biochem. 51, 109-115 (2012).

64. Bassil, E., Coku, A. \& Blumwald, E. Cellular ion homeostasis: Emerging roles of intracellular NHX Na+/H+ antiporters in plant growth and development. J. Exp. Bot. 63, 5727-5740 (2012).

65. Apse, M. P., Aharon, G. S., Snedden, W. A. \& Blumwald, E. Salt tolerance conferred by overexpression of a vacuolar Na+/H+ antiport in Arabidopsis. Science 285, 1256-1258 (1999).

66. Abdelaziz, M. E. et al. The endophytic fungus Piriformospora indica enhances Arabidopsis thaliana growth and modulates $\mathrm{Na}+/ \mathrm{K}+$ homeostasis under salt stress conditions. Plant Sci. 263, 107-115 (2017).

67. Munns, R. et al. Energy costs of salt tolerance in crop plants. New Phytol. 225, 1072-1090 (2020)

68. Shabala, S., Chen, G., Chen, Z. H. \& Pottosin, I. The energy cost of the tonoplast futile sodium leak. New Phytol. 225, 1105-1110 (2020).

69. Apse, M. P. \& Blumwald, E. Na ${ }^{+}$transport in plants. FEBS Letters 581, 2247-2254 (2007).

\section{Acknowledgements}

We acknowledge the logistic support of the Chilean Antarctic Institute (INACH). This study was supported by project FONDECYT 1181034 and REDES 180069.

\section{Author contributions}

M.A.M.-M. and C.T.-D. designed the experiments. M.A.M.-M., I.S.A.-R. and C.T.-D. performed the experiments. M.A.M.-M., I.S.A.-R., P.E.G. and I.D. analyzed and discussed the data. M.A.M.-M. wrote the paper along with I.S.A.-R., C.T.-D., P.E.G. and I.D. All authors reviewed the manuscript.

\section{Competing interests}

The authors declare no competing interests.

\section{Additional information}

Supplementary information is available for this paper at https://doi.org/10.1038/s41598-020-62544-4.

Correspondence and requests for materials should be addressed to M.A.M.-M.

Reprints and permissions information is available at www.nature.com/reprints.

Publisher's note Springer Nature remains neutral with regard to jurisdictional claims in published maps and institutional affiliations.

(c) (i) Open Access This article is licensed under a Creative Commons Attribution 4.0 International

License, which permits use, sharing, adaptation, distribution and reproduction in any medium or format, as long as you give appropriate credit to the original author(s) and the source, provide a link to the Creative Commons license, and indicate if changes were made. The images or other third party material in this article are included in the article's Creative Commons license, unless indicated otherwise in a credit line to the material. If material is not included in the article's Creative Commons license and your intended use is not permitted by statutory regulation or exceeds the permitted use, you will need to obtain permission directly from the copyright holder. To view a copy of this license, visit http://creativecommons.org/licenses/by/4.0/.

(c) The Author(s) 2020 